\title{
Kidney injury by cyclosporine $A$ is aggravated in heme oxygenase-1 deficient mice and involves regulation of microRNAs
}

\author{
Agnieszka Loboda ${ }^{\circledR}$, Olga Mucha1, Paulina Podkalicka1, Mateusz Sobczak', Anna Miksza- \\ Cybulska1, Patrycja Kaczara1, Alicja Jozkowicz' ${ }^{1}$ and Jozef Dulak ${ }^{1,2}$
}

'Department of Medical Biotechnology, Faculty of Biochemistry, Biophysics and Biotechnology, Jagiellonian University, Kraków, Poland; ${ }^{2}$ KardioMed Silesia, Zabrze, Poland

Cyclosporine A (CsA), a widely used immunosuppressive drug, exerts nephrotoxic activities, as demonstrated by increased tubulointerstitial fibrosis, inflammation and podocyte damage. Recently, a number of microRNAs expressed in the kidney have been reported to be elevated during renal damage. Our aim was to investigate the effect of CsA on selected microRNAs in the mouse kidney after CsA treatment. Moreover, as heme oxygenase-1 (HO-1, encoded by the Hmox1 gene) was shown to play a protective role during kidney disorders, we assessed whether HO-1 deficiency in vivo influences the CsA-regulated microRNAs' expression. We have observed that the pro-fibrotic miR-21 and pro-apoptotic miR-34a expression was upregulated in kidneys of $\mathrm{HO}-1$ deficient mice and it was further enhanced by CsA. Concomitantly, the level of anti-fibrotic microRNAs, belonging to miR-29 and miR-200 families, was down-regulated after CsA treatment. Generally, Hmox 1 knock-out (Hmox $\left.1^{-1-}\right)$ animals were more susceptible to CsA treatment, as the mortality rate was 4 out of $9 \mathrm{Hmox}^{-1-}$ mice, and increased fibrosis (Tgfb2, Pai1), inflammation (II6) and apoptosis (Cdkn1ap21) were noticed in the HO-1 deficient kidneys. In summary, our data demonstrate that CsA induces significant changes in the expression of renal microRNAs and emphasize HO-1 deficiency as an important factor contributing to the CsA-mediated renal toxicity.

Key words: cyclosporine A, nephrotoxicity, microRNAs, heme oxygenase-1, HO-1, Hmox 1

Received: 3 September, 2018; revised: 12 November, 2018; accepted: 13 November, 2018; available on-line: 28 November, 2018

e-mail: agnieszka.loboda@uj.edu.pl

Abbreviations: ALAT, alanine aminotransferase; ASPAT, aspartate aminotransferase; $B$ UN; blood urea nitrogen; $C S A$, cyclosporine $A_{i}$ HO-1, heme oxygenase-1; OTA, ochratoxin A; TGF- $\beta 1$, 2, transforming growth factor- $\beta 1,2$

\section{INTRODUCTION}

Cyclosporine A (CsA), a calcineurin inhibitor, is one of the most effective and widely used immunosuppressants in solid organ transplantation and autoimmune diseases; however, it exerts strong nephrotoxic effects. Inflammation, apoptosis, and fibrosis associated with oxidative stress play a pivotal role in producing structural and functional kidney impairment in CsAinduced renal injury (Bennett et al., 1996; Camilleri et al., 2016; Naesens et al., 2009). Recently, modulation of microRNAs' expression has been suggested as a possible new mechanism of action of nephrotoxic agents.
Previously, we have observed increased expression of miR-21 and miR-34a in the mouse kidney after ochratoxin A (OTA) stimulation, a factor with known nephrotoxic activity (Loboda et al., 2017a; Loboda et al., 2017b). The role of microRNAs in CsA nephrotoxicity has been also evaluated in other recent studies showing, among others, up-regulation of pro-fibrotic miR-21 both, in vitro (Chen et al., 2015) and in vivo (Gooch et al., 2017).

Heme oxygenase-1 (HO-1, encoded by the Hmox1 gene) not only catalyzes conversion of heme to biliverdin, carbon monoxide and iron ions, but it also exerts anti-inflammatory, anti-apoptotic and anti-oxidant properties (Loboda et al., 2016a). The level of HO-1 expression in human population can vary because of the known HMOX1 promoter polymorphism - the number of guanosine thymidine dinucleotide $(\mathrm{GT})_{\mathrm{n}}$ repeats in the promoter region is inversely associated with HMOX1 mRNA expression and HO enzyme activity (Loboda et al., 2008; Taha et al., 2010). Importantly, HO-1 was shown to play a pivotal role in the kidney protection against harmful insults in several models of kidney disorders, including unilateral ureteral obstruction (UUO) (Correa-Costa et al., 2010), diabetic induced-glomerular injury (Elmarakby et al., 2012) and transplant rejection (Li et al., 2011). We have already demonstrated that HO-1 protects against OTA-induced nephrotoxicity, as lack of HO-1 increased inflammation, apoptosis and fibrosis, whereas its induction with cobalt protoporphyrin (CoPPIX) halted OTA toxicity (Loboda et al., 2017a).

The importance of HO-1 in CsA-induced kidney failure was already suggested (Shin et al., 2010); however, the involvement of microRNAs in such a process has not been evaluated until now. However, we have previously found that HO-1 is a regulator of microRNAs expression and biogenesis in satellite cells (Kozakowska et al., 2012), it controls miR-206-dependent myogenic program in rhabdomyosarcoma (Ciesla et al., 2016) and modulates miR-378 effect in human non-small cell lung carcinoma (NSCLC) development (Skrzypek et al., 2013). More importantly, there is a cross-talk between HO-1 and microRNAs in the kidney cells (Stachurska et al., 2013).

As HO-1 may regulate the expression of microRNAs and it may exert protective effect against CsA-induced kidney damage, we aimed to investigate a possible involvement of microRNAs in CsA toxicity. We used Hmox1 knock-out mice in order to elucidate the role of HO-1 in the observed effects. For our study, we decided to use a relatively low dose of CsA $(15 \mathrm{mg} /$ $\mathrm{kg} / \mathrm{bw})$, based on the literature data and the fact that 
Hmox1 knock-out animals are generally more sensitive to various stressors. According to the work by Yadav et al., daily intraperitoneal injection of CsA $(15 \mathrm{mg} / \mathrm{kg} /$ day for 30 days) caused renal dysfunction in Tmbim6 knockout mice (Yadav et al., 2015). In another study, various concentrations of CsA (7.5-30 mg/ kg/day injected i.p for 4 weeks) were used and already the lowest dose was able to exert a profibrotic effect (Lim et al., 2012). In conclusion, we have demonstrated that microRNAs affecting such processes as fibrosis and apoptosis might be regulated by $\mathrm{CsA}$ and, of importance, the effect of immunosuppressant is accelerated in HO-1 deficient mice.

\section{MATERIALS AND METHODS}

Ethics statement. All animal experiments were performed in accordance with the national and European regulations upon approval by the First Local Ethical Committee on Animal Testing at the Jagiellonian University in Krakow. Animals were maintained under a constant $12 \mathrm{~h}$ dark/light cycle at the environmental temperature of $22 \pm 2^{\circ} \mathrm{C}$, and were provided with normal laboratory pellet diet and water ad libitum.

Animals. Experiments were performed on $\mathrm{C} 57 \mathrm{BL} / 6 \times \mathrm{FVB}$ mice with normal level of Hmox1 $\left(H \operatorname{mox} 1^{+/+}\right)$and on littermate mice lacking both Hmox1 alleles $\left(H \operatorname{mox} 1^{-1-}\right)$, of both sexes. The mice were generated from the Hmo $\times 1^{+/-}$breeding pairs kindly gifted by Dr. Anupam Agarwal (Birmingham, AL, USA). Animals were genotyped three weeks after birth by PCR using tail DNA, as described previously (Poss \& Tonegawa, 1997).

Experimental procedure. 6-month-old mice fed a low salt diet $\left(\mathrm{Na}^{+} 0.02 \%\right)$ were injected every other day for 19 days intraperitoneally (i.p.) with $15 \mathrm{mg} / \mathrm{kg}$ bw CsA dissolved in ethanol and diluted in olive oil, producing a working solution $(10 \mathrm{mg} / \mathrm{ml})$. As a control, olive oil with ethanol $(10 \%)$ was used. On the last day of experiment, the mice were sacrificed and blood and kidneys were taken for the downstream analysis.

Analysis of renal and liver markers. Serum level of creatinine, blood urea nitrogen (BUN), alanine aminotransferase (ALAT) and aspartate aminotransferase (ASPAT) were determined in blood by using a standard commercial kit (Spotchem Arkray) according to the manufacturer's instructions with the Spotchem EZ SP-4430 analyzer.

Reverse Transcription and quantitative realtime PCR. Total RNA was isolated by using a modified Chomczynski and Sacchi protocol (Chomczynski and Sacchi, 1987). Kidney fragments were homogenized in $1 \mathrm{ml}$ of QIAzol Total RNA Isolation Reagent by using a TissueLyser (Qiagen). Then, samples were subjected to chloroform extraction and isopropanol precipitation. RNA pellets were dissolved in nucleasefree water and subsequently RNA concentration and purity were determined spectrophotometrically by using NanoDrop 1000 (Thermo Fisher Scientific). RevertAid Reverse Transcriptase (Thermo Fisher Scientific) or NCodeTM VILO ${ }^{\text {TM }}$ miRNA cDNA Synthesis Kit (Invitrogen) were used for reverse transcription, as described earlier (Stachurska et al., 2013). Quantitative real-time PCR was done with the use of SYBR Green PCR Master Mix (Sigma-Aldrich), specific reverse and forward primers, and $30 \mathrm{ng}$ of cDNA, utilizing StepOnePlus ${ }^{\mathrm{TM}}$ Real-Time PCR System (Applied Biosystems). Eef2 (encoding eukaryotic translation elongation factor 2, EF-2) and U6 snRNA were used to normalize the expression of examined mRNAs and microRNAs, respectively.
Preparation of the tissue lysates. Fragments of kidney isolated from each animal were homogenized by using an automatic Tissue Lyser (Qiagen) in ice-cold PBS containing 1\% Triton X-100 and protease inhibitors $(1 \mu \mathrm{g} / \mathrm{ml}$ phenylmethylsulfonyl fluoride, $1 \mu \mathrm{g} /$ $\mathrm{ml}$ leupeptin, and $1 \mu \mathrm{g} / \mathrm{ml}$ aprotinin). Then, the samples were incubated for $30 \mathrm{~min}$ on ice and centrifuged $\left(12000 \times g, 10 \mathrm{~min}, 4^{\circ} \mathrm{C}\right)$. Clear supernatants were collected and total protein concentration was determined by the bicinchoninic acid protein assay kit (Sigma-Aldrich), according to the vendor's protocol. Kidney lysates were subjected to luminex analysis, ELISA and/or Western blotting analysis.

Luminex analysis. Kidney lysates, as well as mouse serum, were used for determination of the cytokine content using multiplex Luminex technology. The concentration of interleukin (IL)-6 and plasminogen activator inhibitor-1 (PAI-1) was measured according to the manufacturer's instructions (Millipore, Billerica, MA, USA). Data were analyzed by using a Flexmap 3D instrument and Luminex xPONENT ${ }^{\circledR} 4.0$ Software and Luminex Analyst Program (Luminex Corporation, Austin, Texas, USA).

Western blotting. Western blotting was performed as described previously (Mucha et al., 2018). Membranes were probed with primary antibodies against HO-1 (ADI-SPA-894, Enzo Life Sciences) and $\alpha$-tubulin (CP06, Calbiochem), overnight at $4^{\circ} \mathrm{C}$. Antibodies were diluted in a blocking buffer $(0.1 \%$ Tween 20 in TBS with $5 \%$ nonfat dry milk). HRP activity was detected using Immobilon Western Chemiluminescent HRP Substrate (WBKLS0500 EMD Millipore).

ELISA for HO-1. HO-1 protein quantification in the kidney lysates was performed by using a Mouse Heme Oxygenase 1 ELISA Kit (ab204524, Abcam) according to the manufacturer's instructions.

Masson's trichrome staining. Paraffin-embedded kidney sections $(6 \mu \mathrm{m})$ were sliced, deparaffinized and rehydrated. Kidney fibrosis was assessed with the Masson's trichrome stain (Sigma-Aldrich). Results were observed under light microscopy. Evaluation of fibrosis was performed by a person that was blind to the mice genotype and treatment, using the following assessment - 0 - no signs of collagen deposition; 1 - any sign of collagen deposition; 2 - clearly visible collagen deposition; 3 - collagen deposition on half of field of view; 4 - fibrosis took up more than half of field of view.

Statistical analysis. Data are presented as mean \pm standard error (SEM). Statistical assessment was done by analysis of variance (ANOVA), followed by a Bonferroni post-hoc test for multiple comparisons, or with Student's t-test for two group comparisons. Differences were accepted as statistically significant at $p<0.05$.

\section{RESULTS}

\section{CsA exerts nephrotoxic effects in HO-1 deficient mice}

At first, we confirmed our animal model (HO-1 deficient mice) by assessing the expression of HO-1 and we investigated the effect of CsA treatment on HO-1 expression in the kidneys of wild type animals. We were not able to detect HO-1 in Hmox1 knock-out animals (not shown) and we did not observe any effect of CsA treatment on HO-1 expression in W'T mice (Fig. 1A-C).

Importantly, we have demonstrated that Hmox1 knock-out mice were more susceptible to CsA toxicity. Accordingly, CsA treatment $(15 \mathrm{mg} / \mathrm{kg}$ bw delivered 
A

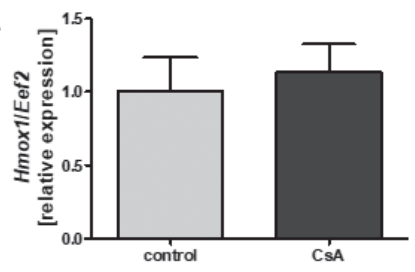

D

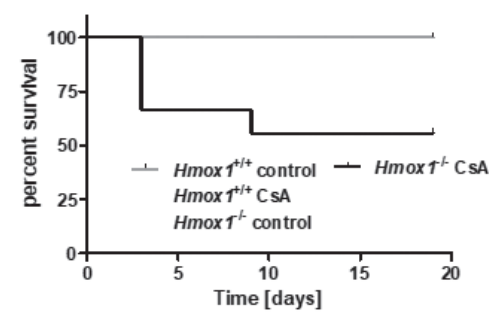

$\mathbf{F}$

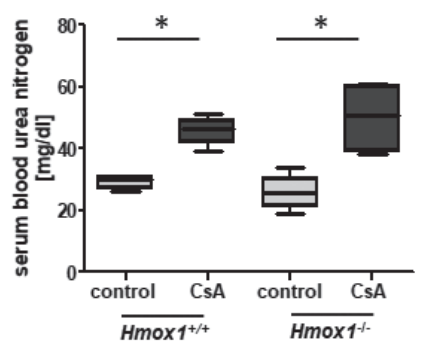

G
B

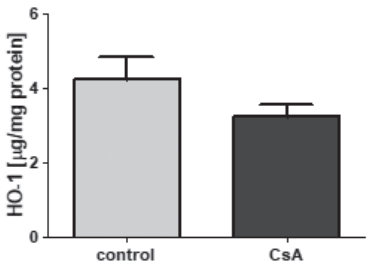

C

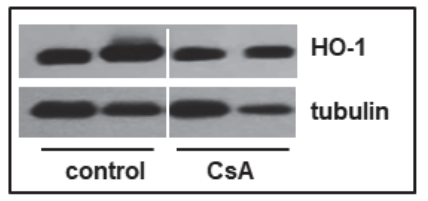

E

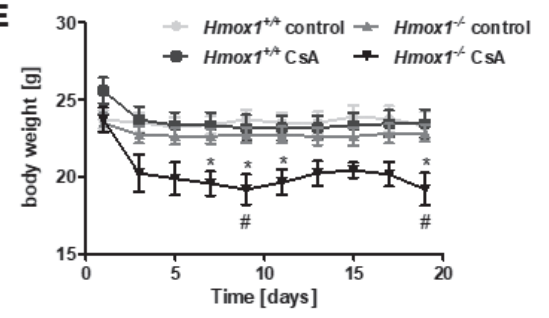

H

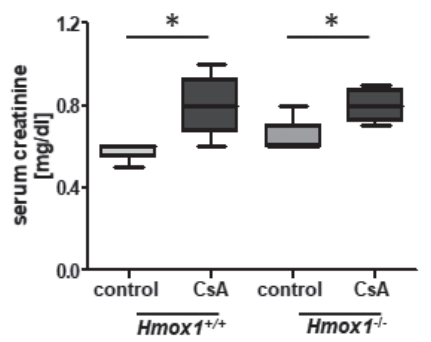

Figure 1. HO-1 expression is not regulated by CsA in wild type animals but Hmox 1 knock-out (Hmox $1^{-/-)}$mice are more susceptible to CsA toxicity.

Hmox1 mRNA expression assessed by real-time PCR (A), protein level assessed by ELISA (B) and western blot (C) in the mouse kidneys

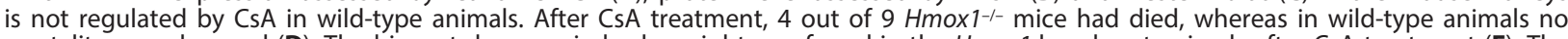
mortality was observed (D). The biggest decrease in body weight was found in the Hmox1 knock-out animals after CSA treatment (E). The markers of renal function, blood urea nitrogen $(\mathbf{F})$ and creatinine level $(\mathbf{G})$ were upregulated in mice of both genotypes, whereas total protein level $(\mathbf{H})$ was increased only in the Hmox 1-1- mice treated with CsA. Mean of 5-9 animals/group, ${ }^{*} p<0.05$ vs. control.

A

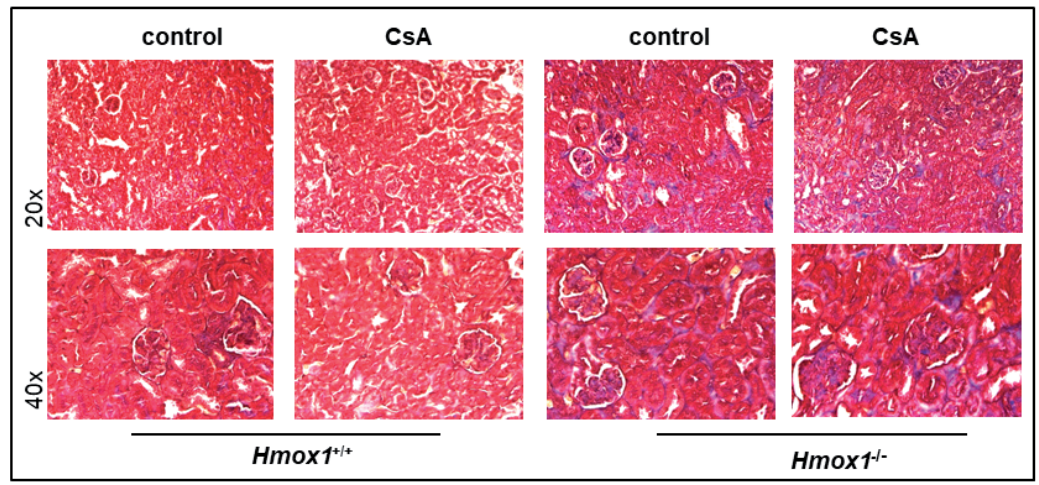

B

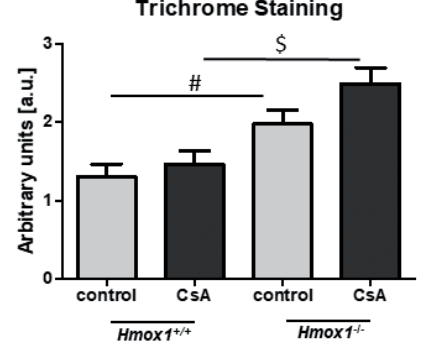

C

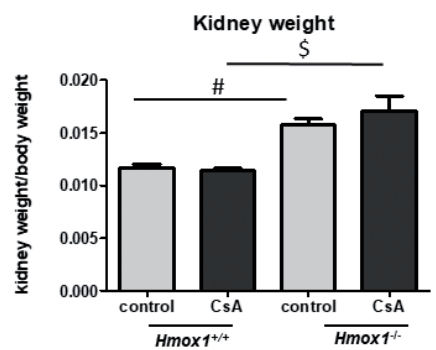

Figure 2. Increased kidney fibrosis is observed in Hmox 1 knock-out animals

Increased collagen deposition (assessed by Masson's trichrome staining) was observed in kidneys of Hmox1 knock-out animals as indicated by the photos (A) and quantification of the staining (B). The difference in kidney weight was also prominent in the Hmox1 knock-out animals (C). Mean of 5-9 animals/group, $\# p<0.05$ vs. Hmox $1^{+++}$control animals, $\$ p<0.05$ vs. Hmox $1^{+/+}$CsA-treated mice. 
A

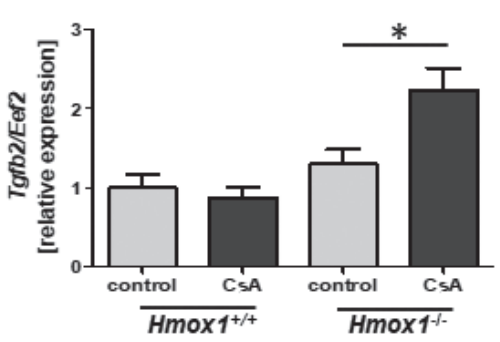

C

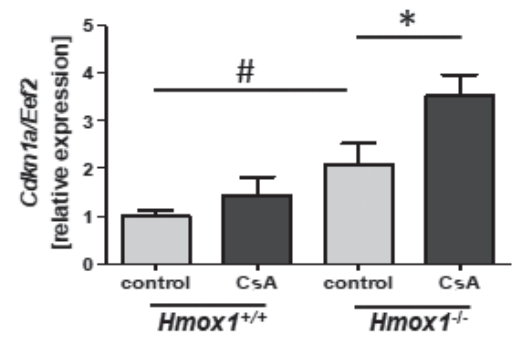

E

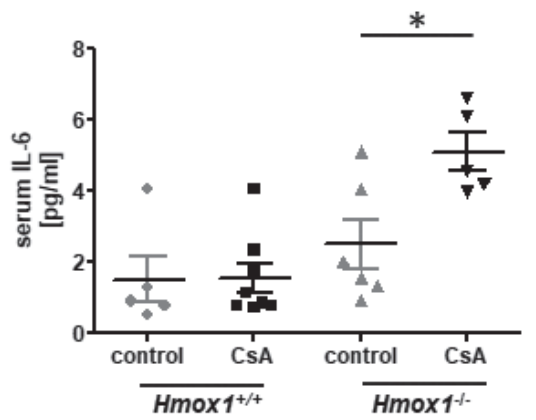

B

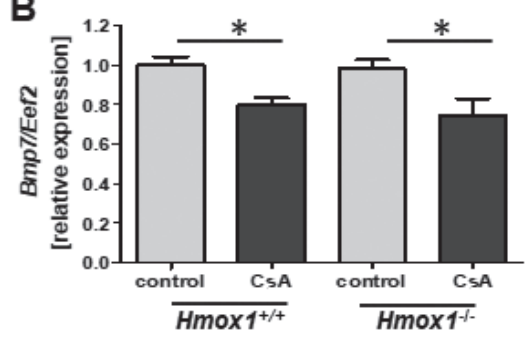

D

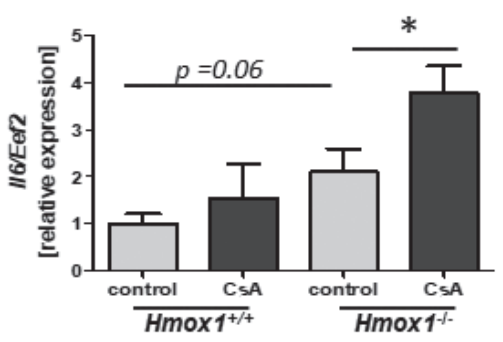

F

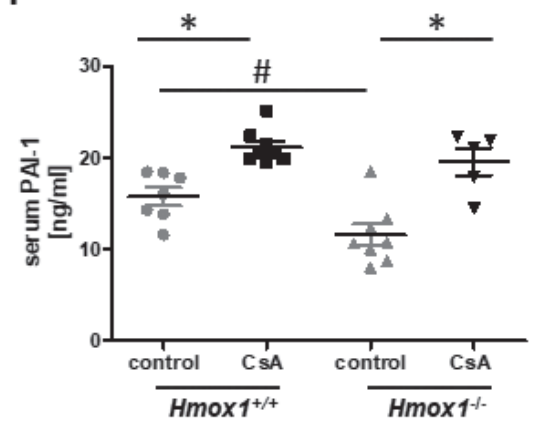

Figure 3. CsA treatment influences the expression of fibrotic, apoptotic and inflammatory markers in the kidneys of $\mathrm{Hmox}^{+/+}$and Hmox $1^{--}$mice.

Pro-fibrotic Tgfb2 (A), anti-fibrotic Bmp7 (B), pro-apoptotic Cdkn1a (C) and pro-inflammatory II6 (D) transcript levels (normalized to Eef2) were assessed using qRT-PCR. Protein level of IL-6 (E) and PAl-1 (F) was checked by luminex in the mouse serum. Data are presented as a fold change over control Hmox $1^{+/+}$mice. Mean of $5-9$ animals/group, ${ }^{*} p<0.05$ vs. control in each genotype, \#p<0.05 vs. Hmox $1^{+/+}$control animals.

intraperitoneally every other day for 19 days) resulted in death of 4 out of 9 Hmox $1^{-/-}$mice ( $44 \%$ mortality) (Fig. 1D). Moreover, CsA caused the highest decrease in the total body weight in HO-1 deficient mice (Fig. 1E). The levels of kidney function markers, namely BUN (Fig. 1F) and creatinine (Fig. 1G) were up-regulated in the mouse serum of both, the Hmox1 $1^{+/+}$and Hmox1/- mice, whereas the total protein level (Fig. 1H) was increased in a statistically significant way only in the Hmox1 knock-out animals treated with CsA. In contrast, markers of the liver injury (ALAT and ASPAT) were not changed (data not shown).

\section{Markers of fibrosis, inflammation and apoptosis are elevated after CsA treatment mostly in mice lacking HO-1}

CsA treatment may lead to fibrosis, inflammation and apoptosis (1). In our experimental model, fibrosis (collagen deposition) assessed by Trichrome staining (Fig. 2A, B) was mostly affected by lack of HO1. Also, the kidney weight was increased in Hmox1 knock-out animals (Fig. 2C), but was not influenced by CsA treatment. However, a more detailed molecular analysis indicated elevated expression of pro-fibrotic transforming growth factor- $\beta 2$ (Tgfb2) in the Hmox1-
/- animals (Fig. 3A), whereas no statistically significant difference in the expression of $T g f b 1$ after CsA treatment was observed (not shown). On the other hand, the level of anti-fibrotic Bmp7 was decreased irrespectively of the mice genotype (Fig. 3B). Expression of Cdkn1a (p21), a p53-inducible gene (Georgakilas et al., 2017) was significantly increased by $\mathrm{CsA}$ in $\mathrm{HO}-1$ deficient mice (Fig. 3C). Similarly, Il6 mRNA expression (Fig. 3D) and IL-6 protein synthesis (Fig. 3E) were increased mostly in the Hmox $1^{1-}$ mice. Interestingly, in the mouse serum, a higher level of plasminogen activator inhibitor-1 (PAI-1) induced by CsA was detected (Fig. 3F), independently of HO-1 presence.

\section{CsA regulates expression of microRNAs in an HO-1 dependent manner}

Based on the importance of some microRNAs in the kidney pathophysiology and on our previous studies with nephrotoxic agent, OTA (Loboda et al., 2017b; Loboda et al., 2016b; Metzinger-Le Meuth et al., 2018), we have assessed if CsA modulates expression of selected microRNAs in the mouse kidney and if the HO-1 level has any impact on such regulation. As indicated in Fig. 4A, B, the level of anti-fibrotic microRNAs, namely miR-29b and miR-200c, was slightly decreased 
A

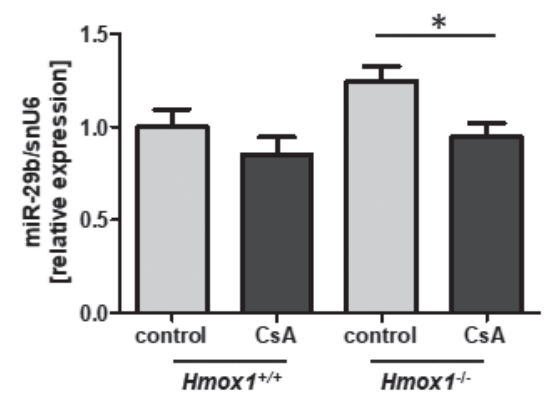

C

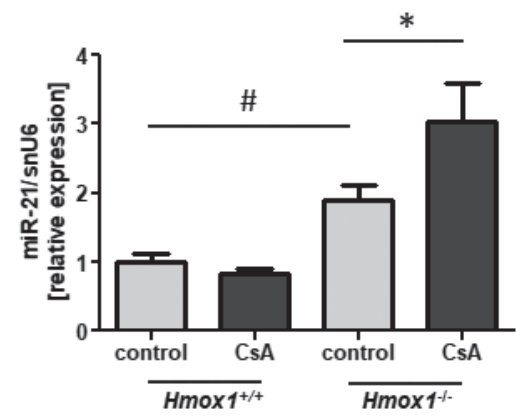

B

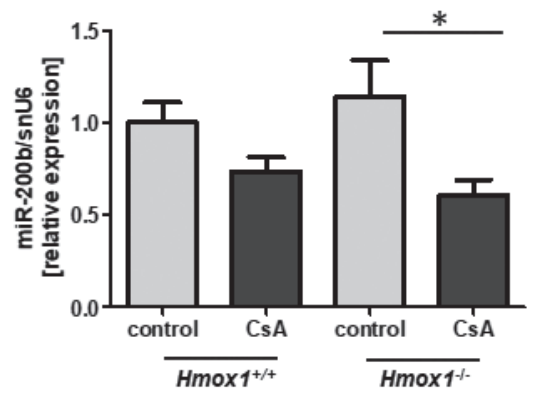

D

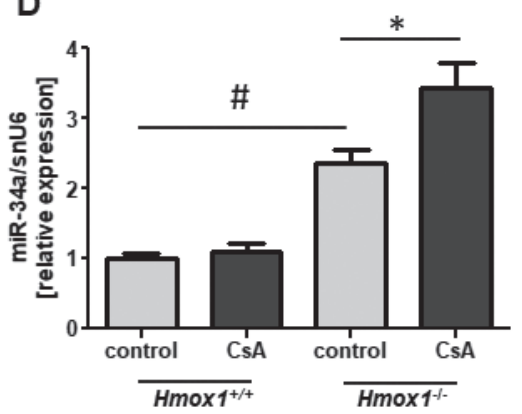

Figure 4. CsA treatment influences expression of microRNAs in the mouse kidneys in an HO-1 dependent way.

Expression of anti-fibrotic miR-29b (A) and miR-200b (B) is decreased in Hmox 1 knock-out animals treated with CsA. Expression of major pro-fibrotic microRNAs, miR-21 (C) and miR-34a (D) is increased by HO-1 deficiency and is further up-regulated by CsA. Transcript levels were normalized to snU6 and is shown as a fold of change over control Hmox $1^{+/+}$mice. Mean of 5-9 animals/group, ${ }^{*} p<0.05$ vs. control in each genotype, $\# p<0.05$ vs. Hmox $1^{+/+}$control animals.

by CsA in WT animals, but the effect was greater (and statistically significant) in mice lacking HO-1. Moreover, the expression of pro-fibrotic miR-21 and pro-apoptotic miR-34a was increased in the kidneys of Hmox1-1mice in comparison to W'T counterparts. Notably, CsA treatment further increased their level only in the Hmox1 knock-out animals (Fig. 4C, D).

\section{DISCUSSION}

Cyclosporine A (CsA)-induced glomerulosclerosis and tubulointerstitial fibrosis is a well-described side effect of CsA treatment that limits its long-term use (Bennett et al., 1996). The mechanisms of CsA toxicity have been investigated for the past several years and new possible factors implicated in its nephrotoxic effect have been demonstrated (Camilleri et al., 2016; Naesens et al., 2009). The aim of our study was to evaluate the involvement of HO-1 in the effects triggered by CsA in mice. Moreover, we have studied contribution of small non-coding RNA molecules - microRNAs in CsA-induced nephrotoxicity.

We and others have reported that an elevated level of HO-1 exerts protective effects under various stressful conditions (reviewed in: Loboda et al., 2016a; Loboda et al., 2008) and previous studies have shown induction of HO-1 after CsA treatment, both in vitro in the rat tubular epithelial NRK-52E cells (Shin et al., 2010) and in vivo in the rat kidney (Capasso et al., 2008). In our hands, no differences in the HO-1 level upon CsA treatment were detected, possibly because of the different experimental scheme with a relatively low dose of CsA used. Nonetheless, as shown by us, the lack of HO-1 causes accumulation of collagen and increased kidney weight (kidney hypertrophy). Moreover, HO-1 deficiency promoted expression of miR-21 and miR-
$34 \mathrm{a}$, the markers of fibrosis and apoptosis, indicating the importance of this protective enzyme in the kidney homeostasis. Such regulation was also observed by us previously in other experimental settings in the mouse kidney (Loboda et al., 2017a) and in fibroblasts isolated from the Hmox1 knock-out mice (Stepniewski et al., 2018). Through its anti-apoptotic, anti-inflammatory and anti-oxidant properties, HO-1 may also protect against toxic insults as the extent of fibrosis was accelerated in the HO-1 knock-out animals treated with OTA (Loboda et al., 2017a; Stachurska et al., 2013).

Interestingly, we have identified several microRNAs that are regulated in the kidneys exposed to OTA, but this effect was HO-1-independent (Loboda et al., 2017a). In contrast, as shown in the study presented here, the differences in the expression of pro-fibrotic miR-21, anti-fibrotic miR-29b and miR-200b, as well as pro-apoptotic miR-34a, were aggravated by the lack of HO-1 in the CsA-induced nephrotoxicity. Notably, miR-21 was previously found to mediate CsA nephrotoxicity via the PTEN/AKT signaling pathway in the human proximal tubular epithelial cells (HPTECs) (Chen et al., 2015). Recent study by Gooch et al. revealed 76 differentially expressed microRNAs in kidneys of mice that received CsA $(20 \mathrm{mg} / \mathrm{kg})$ or vehicle treatment daily for six weeks (Gooch et al., 2017). Except changes in particular microRNAs which were previously linked to renal fibrosis, like let-7d, miR-21, miR-29, miR-30, miR-130, miR-192, and miR-200a, microRNAs that have not been reported to be related to nephrotoxicity or immunosuppression (such as miR-186 and miR-709) were also found to be dysregulated (Gooch et al., 2017). Interestingly, we did not observe induction of miR-21 in the wild type animals. However, even upon more frequent and longer administration of CsA applied by Gooch et al., induction 
of miR-21 in healthy wild type mice was rather moderate (less than 2 fold induction was measured by quantitative real-time PCR analysis and 2.19 fold induction by RNA sequencing). miR-21 was suggested to be a universal biomarker of kidney pathology and was found to be increased in response to various stimuli, not only in this organ but also in the serum, plasma, and urine (reviewed in: Loboda et al., 2016b). Thus, it would be of interest to further extend our analysis beyond the kidney to verify its utility as a biomarker in our experimental scheme.

In our study, we also observed an increased expression of miR-34a, regulated by the p53 signaling pathway, in the CsA-treated HO-1 knock-out mice. DNA damage and cell cycle arrest accompanied with elevated p53 level are found to be early events in the CsA nephrotoxicity, both in vitro (Lally et al., 1999) and in vivo (Shihab et al., 1999). We did not assess expression of p53 or p21 regulator in this study, but we have shown previously that treatment the with another nephrotoxic agent, OTA, leads to a significant and concomitant upregulation of miR-34a, p53, p21 and p53 upregulated modulator of apoptosis (PUMA) expression (Loboda et al., 2017a). Increased apoptosis, including p21 induction, was already observed in the renal proximal tubular cells in vitro after CsA stimulation (Jennings et al., 2007).

In addition, Yuan et al. pointed out to miR-494 as responsible for CsA-induced nephrotoxicity and epithelial to mesenchymal transition (EMT) progression (Yuan et al., 2015). Finally, Sun and coworkers (Sun et al., 2017) identified miR-181c as a factor protecting renal tissues from CsA-induced nephrotoxicity.

Our results indicate that selected microRNAs, which were known to be altered in the diseased kidneys, are regulated by $\mathrm{CsA}$ in an $\mathrm{HO}-1-d e p e n d e n t$ way. It would be also interesting to study if CsA may influence the total pool of microRNAs, as well as the expression of microRNAs processing enzymes. In microRNAs biogenesis, DiGeorge syndrome critical region gene 8, (DGCR8), a heme-dependent protein, plays a critical role in the first step of primary microRNAs (pri-miRNAs) processing (Faller et al., 2007). In our previous study, DGCR8 was shown to be decreased by HO-1 overexpression (Kozakowska et al., 2012), whereas its level was upregulated in porcine renal epithelial cells stimulated by OTA, which concomitantly inhibited HO-1 expression (Stachurska et al., 2013). Therefore, it might be suggested that the DGCR8 level, as well as its properties, depend on the HO-1 activity.

HO-1 is a well-known anti-inflammatory factor (Loboda et al., 2016a, 2008). In the study presented here, expression of IL-6, a marker of inflammation, was induced by CsA at the mRNA level in the kidneys and at the protein level in the serum only in the Hmox 1 -animals, suggesting that the lack of HO-1 triggers an inflammatory reaction after CsA treatment. Similarly, Cdkn1a (p21) expression was elevated only in the HO-1 deficient kidneys.

PAI-1, not expressed in the kidneys under normal conditions, is rapidly induced in a wide variety of acute and chronic renal diseases. Accordingly, PAI-1 was shown to be implicated in glomerulosclerosis and tubulointerstitial fibrosis (reviewed in: (Eddy, 2009). Moreover, CsA-induced up-regulation of PAI-1 was observed in vitro in the rat mesangial cells (Waiser et al., 2002) or in vivo in the rat kidney (Pereira et al., 2006). Here, we report that in Hmox1 knock-out animals, the rate of induction of Pai1 expression after CsA is more pronounced than in wild-type mice $(69 \%$ vs $34 \%$ induction, $p=0.0098)$. Concomitantly, increased collagen deposition and expression of $T g f b 2$ demonstrates pro-fibrotic changes in the kidneys of HO-1 deficient mice subjected to CsA treatment.

Lack or only mild changes in the expression of examined genes in control mice after CsA treatment may suggest that our scheme of the experiment, including the chosen dose of CsA, was too gentle to cause potent fibrotic, inflammatory or apoptotic effects; however, it was sufficient to trigger such a response in mice lacking HO-1.We have also observed mortality in Hmox1 knockout animals after CsA injections, which might indicate (but not prove) an increased sensitivity to nephrotoxic insults. Hence, HO-1 seems to play a protective role against CsA-mediated toxicity in wild-type animals.

Altogether, our data indicate a higher renal damage in animals lacking HO-1. Based on these results, it might be proposed that in HO-1 deficient mice the disruption of kidney architecture might be related to disturbances in extracellular matrix synthesis and increased apoptosis and inflammation, which collectively can be considered as major mechanisms of CsA toxicity. As highlighted in this study, specific microRNAs are also implicated in this process.

It has to be emphasized that our results indicate an increased sensitivity of Hmox1 knock-out animals to CsA treatment, however, in the human population, instead of total HO-1 deficiency, a rather decreased level of HMOX1 mRNA expression and HO enzyme activity might be detected in people with longer $(\mathrm{GT})_{\mathrm{n}}$ repeats in the HMOX1 promoter (Loboda et al., 2008; Taha et al., 2010). Thus, HO-1 heterozygous mice potentially could be used to recapitulate, at least partially, the situation observed in the human population. Nevertheless, one may speculate that CsA toxicity and its influence on microRNAs' expression might be patient-specific. However, such data are not available so far.

Our results may also help to understand some molecular aspects of CsA-related toxicity after heart transplantations. Triple therapy based on calcineurin inhibitors (CsA or tacrolimus), antiproliferative agents (azathioprine or mycophenolate mofetil) and steroids, can be used as the gold standard immunosuppressive therapy after cardiac transplantation (Guethoff et al., 2013; O’Neill et al., 2004). CsA monotherapy has been also tested and its effectiveness was compared with the double therapy (CsA with azathioprine) in the long-term heart transplant recipients, surviving more than 5 years (Sansone et al., 2010). No differences in the survival rate and major events, including heart failure, malignancy, infection and cardiac allograft vasculopathy (CAV), were reported, suggesting the usefulness of CsA treatment (Sansone et al., 2010). Nonetheless, data from retrospective analysis indicate that the renal failure is a frequent cause of death after heart transplantation, and 10 years after such operation around $6 \%$ of patients require dialysis and about $4 \%$ need renal transplantation (reviewed in: (Söderlund \& Rådegran, 2015)). Those adverse effects could be the result of CsA toxicity, therefore, minimizing the use of this immunosuppressant is suggested to decrease chronic kidney diseases after heart transplantations. Recently, involvement of specific microRNAs in the toxic effects of CsA on cardiomyocytes has been also reported. Zhu and coworkers (Zhu et al., 2016) have identified miR-377 as the factor responsible for cardiomyocyte apoptosis after CsA treatment. More importantly, miR-21 level that was increased in the renal tissues after CsA treatment, as shown in our and other studies (Chen et al., 2015), was suggested to be a predictive marker of the development of acute kidney injury after cardiac surgery (Gaede et al., 2016). 
In summary, we have shown that Hmox1 knock-out mice are more susceptible to CsA toxic effects through the increased fibrosis, inflammation and apoptosis, including microRNA regulation. Therefore, the level of Hmox1 expression (which can vary because of the known HO-1 promoter polymorphism) might be an important factor regulating patient's response to the CsA treatment, however, this requires further studies.

\section{Conflict of interest statement}

The authors declare that there is no conflict of interests.

\section{Acknowledgements}

The staff from the animal facility, Karolina Hajduk and Ewa Werner, are acknowledged for excellent technical support and mice breeding.

\section{Acknowledgements of Financial Support}

This work was supported by the Polish Ministry for Science and Higher Education (IP2011 031071) and by the National Centre for Research and Development (STRATEGMED 2/269415/11/NCBR/2015). The Faculty of Biochemistry, Biophysics and Biotechnology of the Jagiellonian University is a partner of the Leading National Research Center (KNOW) supported by the Ministry of Science and Higher Education.

\section{REFERENCES}

Bennett WM, DeMattos A, Meyer MM, Andoh T, Barry JM (1996) Chronic cyclosporine nephropathy: the Achilles' heel of immunosuppressive therapy. Kidney Int 50: 1089-1100

Camilleri B, Bridson JM, Halawa A (2016) Calcineurin inhibitor-sparing strategies in renal transplantation: where are we? a comprehensive review of the current evidence. Exp Clin Transplant 14: 471-483. doi.org/10.6002/ect.2015.0283

Capasso G, Di Gennaro CI, Della Ragione F, Manna C, Ciarcia R, Florio S, Perna A, Pollastro, RM, Damiano S, Mazzoni O, Galletti P, Zappia V (2008) In vivo effect of the natural antioxidant hydroxytyrosol on cyclosporine nephrotoxicity in rats. Nephrol Dial Transplant 23: 1186-1195. doi.org/10.1093/ndt/gfm784

Chen J, Zmijewska A, Zhi D, Mannon RB (2015) Cyclosporine-mediated allograft fibrosis is associated with micro-RNA-21 through AKT signaling. Transpl Int 28: 232-245. doi.org/10.1111/tri.12471

Chomczynski P, Sacchi N (1987) Single-step method of RNA isolation by acid guanidinium thiocyanate-phenol-chloroform extraction. Anal Biochem 162: 156-159. doi.org/10.1006/abio.1987.9999

Ciesla M, Marona P, Kozakowska M, Jez M, Seczynska M, Loboda, A., Bukowska-Strakova, K., Szade A, Walawender, M., Kusior, M., Stepniewski J., Szade, K., Krist, B., Yagensky, O., Urbanik A, Kazanowska B, Dulak J, Jozkowicz A (2016) Heme oxygenase-1 controls an HDAC4-miR-206 pathway of oxidative stress in rhabdomyosarcoma. Cancer Res 76: 5707-5718. doi.org/10.1158/0008-5472. CAN-15-1883

Correa-Costa M, Semedo P, Monteiro APFS, Silva RC, Pereira RL, Gonçalves GM, Marques GDM, Cenedeze MA, Faleiros ACG, Keller AC, Shimizu MHM, Seguro AC, Reis MA, Pacheco-Silva A, Câmara NOS (2010) Induction of heme oxygenase-1 can halt and even reverse renal tubule-interstitial fibrosis. PloS One 5: e14298. doi.org/10.1371/journal.pone.0014298

Eddy AA (2009) Serine proteases, inhibitors and receptors in renal fibrosis. Thromb Haemost 101: 656-664.

Elmarakby AA, Faulkner J, Baban B, Saleh MA, Sullivan JC (2012) Induction of hemeoxygenase-1 reduces glomerular injury and apoptosis in diabetic spontaneously hypertensive rats. Am J Physiol Renal Physiol 302: F791-F800. doi.org/10.1152/ajprenal.00472.2011

Faller M, Matsunaga M, Yin S, Loo JA, Guo F (2007) Heme is involved in microRNA processing. Nat Struct Mol Biol 14: 23-29. doi.org/10.1038/nsmb1182

Gaede L, Liebetrau C, Blumenstein J, Troidl C, Dörr O, Kim W-K, Gottfried K, Voss S, Berkowitsch A, Walther T, Nef H, Hamm CW, Möllmann H (2016) Plasma microRNA-21 for the early prediction of acute kidney injury in patients undergoing major cardiac surgery. Nephrol Dial Transplant 31: 760-766. doi.org/10.1093/ndt/ gfw007
Georgakilas AG, Martin OA, Bonner WM (2017) p21: A Two-Faced Genome Guardian. Trends Mol Med 23: 310-319. doi.org/10.1016/j. molmed.2017.02.001

Gooch JL, King C, Francis CE, Garcia PS, Bai Y (2017) Cyclosporine A alters expression of renal microRNAs: New insights into calcineurin inhibitor nephrotoxicity. PloS One 12: e0175242. doi.org/10.1371/journal.pone.0175242

Guethoff S, Meiser BM, Groetzner J, Eifert S, Grinninger C, Ueberfuhr P, Reichart B, Hagl C, Kaczmarek I (2013) Ten-year results of a randomized trial comparing tacrolimus versus cyclosporine a in combination with mycophenolate mofetil after heart transplantation. Transplantation 95: 629-634. doi.org/10.1097/TP.0b013e318277e378

Jennings P, Koppelstaetter C, Aydin S, Abberger T, Wolf AM, Mayer G, Pfaller W (2007) Cyclosporine A induces senescence in renal tubular epithelial cells. Am J Physiol Renal Physiol 293: F831-F838. doi.org/10.1152/ajprenal.00005.2007

Kozakowska M, Ciesla M, Stefanska A, Skrzypek K, Was H, Jazwa A, Grochot-Przeczek A, Kotlinowski J, Szymula A, Bartelik A, Mazan M, Yagensky O, Florczyk U, Lemke K, Zebzda A, Dyduch G, Nowak W, Szade K, Stepniewski J, Majka M, Derlacz R, Loboda A, Dulak J, Jozkowicz A (2012) Heme oxygenase-1 inhibits myoblast differentiation by targeting myomirs. Antioxid Redox Signal 16: 113-127. doi.org/10.1089/ars.2011.3964

Lally C, Healy E, Ryan MP (1999) Cyclosporine A-induced cell cycle arrest and cell death in renal epithelial cells. Kidney Int 56: 1254 1257. doi.org/10.1046/j.1523-1755.1999.00696.x

Li S-D, Wang L, Wang K-Y, Liang P, Wu G, Zhang K-Q, Li Q-S, Jin F-S (2011) Heme oxygenase-1 expression and its significance for acute rejection following kidney transplantation in rats. Transplant Proc 43: 1980-1984. doi.org/10.1016/j.transproceed.2011.03.032

Lim SW, Hyoung BJ, Piao SG, Doh, KC, Chung BH, Yang CW (2012) Chronic cyclosporine nephropathy is characterized by excessive autophagosome formation and decreased autophagic clearance. Transplantation 94: 218-225. doi.org/10.1097/TP.0b013e31825ace5c

Loboda A, Damulewicz M, Pyza E, Jozkowicz A, Dulak J (2016a) Role of Nrf2/HO-1 system in development, oxidative stress response and diseases: an evolutionarily conserved mechanism. Cell Mol Life Sci 73: 3221-3247. doi.org/10.1007/s00018-016-2223-0

Loboda A, Jazwa A, Grochot-Przeczek A, Rutkowski AJ, Cisowski J, Agarwal A, Jozkowicz A, Dulak J (2008) Heme oxygenase-1 and the vascular bed: from molecular mechanisms to therapeutic opportunities. Antioxid Redox Signal 10: 1767-1812. doi.org/10.1089/ ars.2008.2043

Loboda A, Sobczak M, Jozkowicz A, Dulak J (2016b) TGF- $\beta 1 /$ Smads and miR-21 in renal fibrosis and inflammation. Mediators Inflamm 2016: 8319283, doi.org/10.1155/2016/8319283

Loboda A, Stachurska A, Podkalicka P, Sobczak M, Mucha O, Witalisz-Siepracka A, Jozkowicz A, Dulak J (2017a) Effect of heme oxygenase-1 on ochratoxin A-induced nephrotoxicity in mice. Int J Biochem Cell Biol 84: 46-57. doi.org/10.1016/j.biocel.2017.01.003

Loboda A, Stachurska A, Sobczak M, Podkalicka P, Mucha O, Jozkowicz A, Dulak J (2017b) Nrf2 deficiency exacerbates ochratoxin A-induced toxicity in vitro and in vivo. Toxicology 389: 42-52. doi.org/10.1016/j.tox.2017.07.004

Metzinger-Le Meuth V, Fourdinier O, Charnaux N, Massy ZA, Metzinger L (2018) The expanding roles of microRNAs in kidney pathophysiology. Nephrol Dial Transplant. doi.org/10.1093/ndt/gfy140

Mucha O, Podkalicka P, Czarnek M, Biela A, Mieczkowski M, Kachamakova-Trojanowska N, Stepniewski J, Jozkowicz A, Dulak J, Loboda A (2018) Pharmacological versus genetic inhibition of heme oxygenase-1 - the comparison of metalloporphyrins, shRNA and CRISPR/Cas9 system. Acta Biochim Pol 65: 277-286. doi.org/10.18388/abp.2017_2542

Naesens M, Kuypers DRJ, Sarwal M (2009) Calcineurin inhibitor nephrotoxicity. Clin J Am Soc Nephrol 4: 481-508. doi.org/10.2215/ CJN.04800908

O’Neill JO, Taylor DO, Starling RC (2004) Immunosuppression for cardiac transplantation--the past, present and future Transplant Proc 36: 309S-313S. doi.org/10.1016/j.transproceed.2004.01.010

Pereira MG, Câmara NOS, Campaholle G, Cenedeze MA, de Paula Antunes Teixeira V, dos Reis MA, Pacheco-Silva A (2006) Pioglitazone limits cyclosporine nephrotoxicity in rats. Int Immunopharmacol. 6: 1943-1951. doi.org/10.1016/j.intimp.2006.07.024

Poss KD, Tonegawa S (1997) Heme oxygenase 1 is required for mammalian iron reutilization. Proc Natl Acad Sci U S A 94: 10919-10924 Sansone F, Boffini M, Comoglio C, Checco L, Saviolo R, Centofanti P, La Torre M, Rinaldi M (2010) Results with cyclosporine monotherapy in long-term cardiac transplant recipients. Transplant Proc. 42: 1291-1293. doi.org/10.1016/j.transproceed.2010.03.126

Shihab FS, Andoh TF, Tanner AM, Yi H, Bennett WM (1999) Expression of apoptosis regulatory genes in chronic cyclosporine nephrotoxicity favors apoptosis. Kidney Int 56: 2147-2159. doi.org/10.1046/j.1523-1755.1999.00794.x

Shin D, Park H-M, Jung K-A, Choi H-G, Kim J-A, Kim D-D, Kim SG, Kang KW, Ku SK, Kensler TW, Kwak M-K (2010) The NRF2-heme oxygenase-1 system modulates cyclosporin A-induced 
epithelial-mesenchymal transition and renal fibrosis. Free Radic Biol Med 48: 1051-1063. doi.org/10.1016/j.freeradbiomed.2010.01.021

Skrzypek K, Tertil M, Golda S, Ciesla M, Weglarczyk K, Collet G, Guichard A, Kozakowska M, Boczkowski J, Was H, Gil T, Kuzdzal J, Muchova L, Vitek L, Loboda A, Jozkowicz A, Kieda C, Dulak J (2013) Interplay between heme oxygenase-1 and miR-378 affects non-small cell lung carcinoma growth, vascularization, and metastasis. Antioxid Redox Signal 19: 644-660. doi.org/10.1089/ ars.2013.5184

Söderlund C, Rådegran G (2015) Immunosuppressive therapies after heart transplantation--The balance between under- and over-immunosuppression. Transplant Rev (Orlando) 29: 181-189. doi.org/10.1016/j.trre.2015.02.005

Stachurska A, Ciesla M, Kozakowska M, Wolffram S, Boesch-Saadatmandi C, Rimbach G, Jozkowicz A, Dulak J, Loboda A (2013) Cross-talk between microRNAs, nuclear factor E2-related factor 2, and heme oxygenase-1 in ochratoxin A-induced toxic effects in renal proximal tubular epithelial cells. Mol Nutr Food Res 57: 504-515. doi.org/10.1002/mnfr.201200456

Stepniewski J, Pacholczak T, Skrzypczyk A, Ciesla M, Szade A, Szade K, Bidanel R, Langrzyk A, Grochowski R, Vandermeeren F, Kachamakova-Trojanowska N, Jez M, Drabik G, Nakanishi M, Jozkowicz A, Dulak J (2018) Heme oxygenase-1 affects generation and spontaneous cardiac differentiation of induced pluripotent stem cells. IUBMB Life 70: 129-142. doi.org/10.1002/iub.1711

Sun W, Min B, Du D, Yang F, Meng J, Wang W, Zhao J, Tan X, Li Z, Sun J (2017) miR-181c protects CsA-induced renal damage and fibrosis through inhibiting EMT. FEBS Lett 591: 3588-3599. doi.org/10.1002/1873-3468.12872

Taha H, Skrzypek K, Guevara I, Nigisch A, Mustafa S, Grochot-Przeczek A, Ferdek P, Was H, Kotlinowski J, Kozakowska M, Balcerczyk A, Muchova L, Vitek L, Weigel G, Dulak J, Jozkowicz A (2010) Role of heme oxygenase-1 in human endothelial cells: lesson from the promoter allelic variants. Arterioscler Thromb Vasc Biol 30: 1634 1641. doi.org/10.1161/ATVBAHA.110.207316

Waiser J, Dell K, Böhler T, Dogu E, Gaedeke J, Budde K, Neumayer $\mathrm{H}-\mathrm{H}$ (2002) Cyclosporine A up-regulates the expression of TGFbeta1 and its receptors type I and type II in rat mesangial cells. Nephrol Dial Transplant. 17: 1568-1577

Yadav RK, Lee G-H, Lee H-Y, Li, B, Jung, H-E, Rashid H-O, Choi MK, Yadav BK, Kim W-H, Kim K-W, Park B-H, Kim W, Lee Y-C, Kim H-R, Chae H-J (2015) TMBIM6 (transmembrane BAX inhibitor motif containing 6) enhances autophagy and reduces renal dysfunction in a cyclosporine A-induced nephrotoxicity model. Autophagy 11: 1760-1774. doi.org/10.1080/15548627.2015.1082021

Yuan J, Benway CJ, Bagley J, Iacomini J (2015) MicroRNA-494 promotes cyclosporine-induced nephrotoxicity and epithelial to mesenchymal transition by inhibiting PTEN. Am J Transplant 15: 16821691. doi.org/10.1111/ajt.13161

Zhu X-D, Chi J-Y, Liang H-H, Huangfu L-T, Guo Z-D, Zou H, Yin X-H (2016) MicroRNA-377 mediates cardiomyocyte apoptosis induced by cyclosporin A. Can J Cardiol 32: 1249-1259. doi.org/10.1016/j.cjca.2015.11.012 